\title{
Perceptions about participation in cancer clinical trials in New York state
}

\author{
Danielle M Crookes, MPH, ${ }^{a}$ Chang Wang, BA, ${ }^{a}$ Karen R DeMairo, BA, MHSA, ${ }^{b}$ \\ Maureen Killackey, MD, ${ }^{\mathrm{c}}$ Jean C LePere, MPH, MS, ${ }^{\mathrm{d}}$ Anita McFarlane, MPH, ${ }^{\mathrm{e}}$ Darryl \\ Somayaji, PhD, RN, ${ }^{\mathrm{f}}$ Patti Allen, BA, ${ }^{\mathrm{g}}$ Linda Bily, MA, ${ }^{\mathrm{h}}$ Beth Ann Brundage, RN, OCN, ${ }^{\mathrm{i}}$ \\ Rosalie Canosa, LCSW-R, MPA, Diana Crean, BS, CCRP, ${ }^{\mathrm{k}}$ and Lina Jandorf, $\mathrm{MA}^{\mathrm{a}}$
}

${ }^{a}$ Department of Oncological Sciences, Icahn School of Medicine at Mount Sinai, New York City; ${ }^{b}$ The Leukemia \& Lymphoma Society, Hauppauge, New York; 'Weill Cornell Medical College, New York City; ${ }^{\mathrm{d}}$ Memorial Sloan-Kettering Cancer Center, New

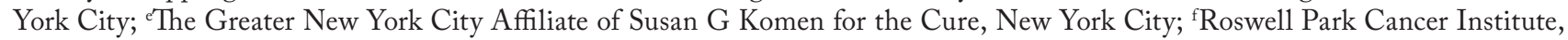

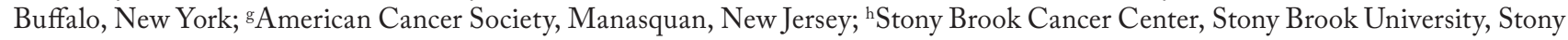

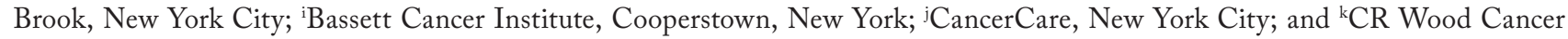
Center at Glens Falls Hospital, Glens Falls, New York

Background Clinical trials are valuable in advancing cancer care through the investigation of ways in which to better prevent, detect and diagnose, and/or treat cancer. Recruitment of adults into clinical trials has historically been low.

Objective To survey adult cancer patients who reside in New York state to better understand their participation in and attitudes about clinical trials.

Methods From January 2012-April 2013, we conducted a one-time survey about clinical trials in 8 cancer-treatment or cancerpatient support organizations in the state. Surveys were offered in person and online to adults with a past or current cancer diagnosis. Analysis was limited to adults who resided in the state and provided a self-reported status of previous participation in clinical trials.

Results Of the 1,832 participants who completed the survey, 1,475 were included in the analysis. Our sample represented all regions of the state. Most of the respondents (68.1\%) had never participated in a clinical trial. Almost $32 \%$ said they had never received information about research studies. Most $(84 \%)$ felt that patients should be asked to participate in clinical trials, but fewer $(70 \%)$ were willing to be approached about participation.

Limitations The sample is predominantly white and female and overrepresents breast and hematologic cancers.

Conclusions Increased outreach coupled with a team approach to educate and enroll patients in clinical trials may be the necessary first steps to increase participation in trials and ensure a diverse sample of participants.

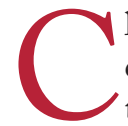

linical trials are valuable in advancing cancer care because they are rigorous studies used to investigate ways in which cancer can be better prevented, detected and diagnosed, and/or treated. The National Cancer Institute lists more than 12,000 clinical trials that are currently recruiting patients, ${ }^{1}$ yet despite that significant number and the benefits of improving patient care based on trial findings, recruitment into clinical trials has historically been low among adults. ${ }^{2}$

The barriers to participation in cancer clinical trials include a variety of patient- and protocol-related factors. ${ }^{3}$ Deterrents of underrepresented groups, such as racial and ethnic minorities and those of low socioeconomic status, include mistrust of the medical community, concern about the extra burden of participation (ie, cost and transportation), and a desire not to feel like they are an experi- ment. ${ }^{4,5}$ Randomization is one of the most common patient-identified, protocol-related barriers to participation. $3,4,6,7$ The patients' concern about receiving either a placebo or treatment that does not offer the best option ${ }^{3}$ and their fears of side effects are significant protocol-related barriers to participation. ${ }^{8}$

Awareness about the availability of a clinical trial, a necessary first step in the consideration to participate in a trial, ${ }^{9}$ also presents a major barrier to participation. Communication between patients and oncologists about clinical trials has been shown to be one of the most important influences in a patient's decision to participate in a trial. Fenton and colleagues ${ }^{10}$ found that $45 \%$ of those who participated in studies did so because their physicians had recommended it, and $60 \%$ of those who had not participated in a trial said that they were unaware of appropriate trials. Similarly, Weckstein and col-

Accepted for publication November 13, 2014. Correspondence: Lina Jandorf, MA; Lina.Jandorf@mssm.edu. Disclosures: The authors have no disclosures to make. JCSO 2015;13:62-72. @2015 Frontline Medical Communications. DOI 10.12788/icso.0110. 
leagues ${ }^{11}$ reported that $44 \%$ of the patients they surveyed who did not participate in a clinical trial could not recall having a conversation about clinical trials with their provider. Receiving information about appropriate clinical trials is an important facilitator to participation. Patients are more inclined to participate in a clinical trial when they are provided more information about the study. ${ }^{6}$ In addition, when patients perceive that their doctors want them to join trials, they may be more likely to accept recruitment into a trial. ${ }^{7}$

Cancer is the second-leading cause of death in New York state and presents an important public health concern for health officials. ${ }^{12}$ Cancer incidence and mortality are recorded through the New York State Cancer Registry, but there is no formal, publicly available resource to track enrollment in clinical trials. The New York State Cancer Consortium (NYSCC) is a statewide coalition of organizations charged with the development and implementation of the state's Comprehensive Cancer Control Plan. This plan is a roadmap to achieve reductions in cancer rates and to improve outcomes for people who have been diagnosed with cancer. The plan includes developmental objectives and strategies for increasing clinical trial enrollment. Although there has been research conducted at the international ${ }^{7}$ and national ${ }^{3,13}$ level on participation in clinical trials, to our knowledge, there has not been a statewide assessment of barriers and facilitators to clinical trial participation in cancer patients in the state of New York. Thus, an NYSCC subcommittee was formed to better understand the attitudes and barriers to clinical trial enrollment in the state. ${ }^{12}$ As part of the process of better understanding those attitudes and participation in clinical trials in the state, we report findings from a survey conducted from January 2012 to April 2013 among adult patients who had ever received a cancer diagnosis and who currently resided in the state.

\section{Methods}

From January 2012 to April 2013, we conducted a onetime survey about clinical trials in adults (aged 18 years or older) who had ever received a cancer diagnosis. The survey consisted of 38 items that captured participant demographics information (15 items), information about cancer diagnosis (2 items), trust in physician (1 item), previous experience with and attitudes about clinical trials (5 items), perceptions about different elements of randomized trials (6 items), barriers and facilitators to participating in a clinical trial (4 items), modes of communication about clinical trials (2 items), and an open-ended question about facilitators for future studies (1 item). Patients who had previously participated in a clinical trial were also asked to rate their clinical trial experience using a 4-point Likert-type scale (1 item) and to provide open-ended feedback about what additional information they would have liked to receive that they did not receive when they participated (1 items). Many of the survey questions were drawn from previous literature ${ }^{10,14}$ or were developed in-house. Data were collected without patients' names or identifying information, with the exception of patients' zip codes.

The survey was administered by 8 organizations in the state: 3 cancer-patient support organizations, 2 academic cancer centers, and 3 community hospitals with oncology programs. Organizations were recruited through networks of the NYSCC. Eligible organizations had to be located within the state, either provide cancer treatment or be a cancer-patient support organization, and had to have a champion who was willing to manage data collection. Internal review board (IRB) approval was initially obtained at the Icahn School of Medicine at Mount Sinai (ISMMS). Each of the coordinating organizations either requested their own IRB approval or received administrative approval to use the ISMMS approval. Waiver of documented signed consent was granted for the conduct of this study, and all participants were provided with a written consent summary prior to completion of the survey.

Based on the needs of the participating organizations, the survey was administered on paper and online. The 5 patient-care organizations administered a paper version of the survey through face-to-face interviews, participant self-completion, and telephone interviews. Recruitment at these organizations took place in cancer-patient care areas such as medical and radiation oncology waiting rooms. Paper surveys were offered in English and Spanish. Faceto-face and telephone interviews were done by either volunteers or staff at each site. The 3 patient support organizations administered an identical online version of the survey using SurveyMonkey. Sites e-mailed the link to existing e-mail contact lists and allowed the participants to fill out the survey anonymously. Because previous communication with listserv and e-mail contacts had always been in English, the online survey was offered in English only.

Data analysis was completed using SPSS version 20. Pearson chi-square and $t$ test statistics were used to measure associations between previous participation in clinical trials and demographics and attitude variables. All of the associations were considered significant at the $5 \%$ significance level.

\section{Results}

During the study period, 1,832 patients completed the survey $-479(26.1 \%)$ on paper and 1,353 (73.9\%) online. Of those, 1,492 (81.4\%) met eligibility criteria (aged 18 years or older, had a cancer diagnosis, currently resided in the state) and were included in the study. Of the eligible 1,492 respondents, 1,475 (98.8\%) answered prompts about their previous participation in research studies and thus were included in this analysis. 


\section{Demographics}

As seen in Table 1, the sample included residents of the state's 11 regions, with most of them residing in New York City (24.7\%), Long Island (17.6\%), Hudson Valley (12.3\%), Finger Lakes (11.9\%), and Central Leatherstocking (10.1\%). The sample was predominantly female (72.7\%), white (85.5\%), non-Latino (94.3\%), and partnered (including married, living with a partner, and partnered but living alone; $71.1 \%$ ). The median age was 57 years (SD, 11.53). Just over half of the sample was employed $(53.6 \%)$, of whom $68.6 \%$ were employed part time. Most respondents (67.5\%) reported an annual income of $\$ 50,000$ or more, and most (75.9\%) had completed some college education or higher. The most common cancer malignancies reported were breast (38.4\%), lymphoma (12.5\%), and leukemia (9.0\%). A comprehensive list of all cancers represented in the sample is provided in Table 1. Most diagnoses (64.1\%) had occurred within the previous 5 years.

Most respondents (86.3\%) reported that in the previous year they sought medical care when they needed to, and $86.1 \%$ reported following doctor's advice and referrals when provided. Nearly $88 \%$ agreed that they trusted their oncologist to put their medical needs above all else. In all, 1,005 of respondents (68.1\%) had never participated in a clinical trial. Participation in clinical trials was associated with gender, race, education level, household income, and time living in the United States. Women were more likely than were men to have participated in research studies $(33.6 \%$ vs $27.6 \% ; P=.03)$. White patients had the highest participation rates $(32.7 \%)$. Participation for blacks was 22.0\%; Hispanics, 12.9\%; and for patients categorized as All Other Races, it was (31.6\%; $P=.021)$. Patients educated at a high school level or below had lower trial participation rates $(27.2 \%)$ than did patients who had completed some level of college education $(30.6 \%)$ or those who had gone on to postgraduate education $(36.8 \% ; P=.009)$. Household economy was significantly associated with participation. Patients with annual household incomes of $\$ 50,000$ or higher were more likely to have participated in studies than were those who reported earning less than $\$ 50,000$ a year (34.4\% vs $26.8 \% ; P=.006)$. Respondents who had lived in the United States all of their lives had higher rates of clinical trial participation than did those who had not lived in the US (33.0\% vs $24.9 \%$; $P=.027)$.

Cancer type was associated with previous participation in a research study $(P=.02$; Table 1$)$. The year of initial cancer diagnosis was also associated with participation in research studies. Participation in clinical trials was lower for those who had been diagnosed in the decades before 2000 (1950s-1989, 33.3\%; 1990s, 35.3\%) than for those who had received a diagnosis between 2000 and 2005 (40.6\%; $P<.001)$. The lowest rate of participation, $27.8 \%$, was among patients who had received their diagnoses most recently (January 2006-April 2013).

\section{Attitudes, barriers, and facilitators to clinical trial participation}

We assessed patients' participation in research studies against their responses to a series of questions about their attitudes toward research studies, contact personnel, and research methods. For the purpose of the analyses, responses to questions were collapsed into Yes, No (including Not Sure), Important (including Somewhat Important), Neutral, or Unimportant (including Somewhat Unimportant).

Tables 2 and 3 summarize data regarding attitudes toward the conduct of clinical trials. Factors that were associated with participation in trials were patients' knowledge of the randomization of studies, the convenience and compensation associated with studies, the administration of drugs, and patients' altruistic feelings toward participation. Patients were assessed on their attitudes toward randomized studies (Table 2). Six questions about various elements of randomization were posed to respondents and were later collapsed into an index measuring positive attitude about randomization. A score of 1 was given to each question answered positively. The highest possible score was 6 (indicating all positive responses) and the lowest was 0 (all negative responses). The mean score was 3.18 (SD, 1.93). Those who had previously participated in studies had a higher index score (mean, 3.59; SD, 1.93) than did those who had not previously participated in a trial (mean, 2.96; SD, 1.93), $t(1,567)=6.027(P<.001)$. Respondents showed an increasing proclivity to have participated in research studies with an increasing positive score from $0-5$, and although there was a decrease in participation rates between scores of 5 and 6, this difference was not statistically significant.

Patient participation in clinical trials was also associated with the convenience of the trial (Table 3). Patients who were indifferent about the number of visits required in a trial (38.6\%) were more likely to have participated in a trial than those who felt that the number of visits was either important (27.2\%) or unimportant (31.2\%; $P=.029)$. Attitudes toward the convenience of scheduling study appointments at the same time as existing medical appointments were also associated with past participation. Patients who thought scheduling study visits at the same time as existing medical appointments was unimportant were more likely to have participated in previous studies than were those who thought it was important or felt neutral about it ( $34.2 \%$ vs $25.3 \%$ vs $29.7 ; P=.01)$.

Patients who had a neutral attitude toward being compensated for their participation in studies were more likely to have previously participated in a study (37.4\%) than those who felt it was important $(28.5 \%)$ or unimportant 
TABLE 1 Characteristics of survey respondents

No. of patients, $\mathrm{n}(\%)$

\begin{tabular}{|c|c|c|c|c|}
\hline \multirow[b]{2}{*}{ Characteristic } & \multirow[b]{2}{*}{$\begin{array}{c}\text { Total } \\
(N=1,475)\end{array}$} & & \multirow[b]{2}{*}{$\boldsymbol{P}$} \\
\hline & & $\begin{array}{c}\text { Have } \\
\text { participated } \\
(n=470)\end{array}$ & $\begin{array}{c}\text { Have not } \\
\text { participated } \\
(n=1,005)\end{array}$ & \\
\hline $\begin{array}{l}\text { Survey mode } \\
\text { Online } \\
\text { Paper }\end{array}$ & $\begin{array}{r}1,082(73.4) \\
393(26.6)\end{array}$ & $\begin{array}{l}365(33.7) \\
105(26.7)\end{array}$ & $\begin{array}{l}717(66.3) \\
288(73.3)\end{array}$ & 011 \\
\hline $\begin{array}{l}\text { Location } \\
\text { Capital-Saratoga } \\
\text { Central Leatherstocking } \\
\text { Chautauqua-Allegheny } \\
\text { Finger Lakes } \\
\text { Hudson Valley } \\
\text { Long Island } \\
\text { New York City } \\
\text { Niagara Frontier } \\
\text { The Adirondacks } \\
\text { The Catskills } \\
\text { Thousand Islands-Sea }\end{array}$ & $\begin{aligned} & 98(6.6) \\
& 149(10.1) \\
& 16(1.1) \\
& 176(11.9) \\
& 182(12.3) \\
& 259(17.6) \\
& 364(24.7) \\
& 115(7.8) \\
& 54(3.7) \\
& 43(2.9) \\
& 19(1.3)\end{aligned}$ & $\begin{array}{r}34(34.7) \\
37(24.8) \\
3(18.8) \\
67(38.1) \\
57(31.3) \\
72(27.8) \\
110(30.2) \\
59(51.3) \\
19(35.2) \\
10(23.3) \\
2(10.5)\end{array}$ & $\begin{array}{r}64(65.3) \\
112(75.2) \\
13(81.2) \\
109(61.9) \\
125(68.7) \\
187(72.2) \\
254(69.8) \\
56(48.7) \\
35(64.8) \\
33(76.7) \\
17(89.5)\end{array}$ & .000 \\
\hline $\begin{array}{l}\text { Age, y } \\
18-39 \\
40-49 \\
50-59 \\
60-64 \\
65 \text { or older }\end{array}$ & $\begin{array}{l}110(7.5) \\
241(16.3) \\
510(34.6) \\
229(15.5) \\
385(26.1)\end{array}$ & $\begin{array}{r}41(37.3) \\
85(35.3) \\
161(31.6) \\
70(30.6) \\
113(29.4)\end{array}$ & $\begin{array}{r}69(62.7) \\
156(64.7) \\
349(68.4) \\
159(69.4) \\
272(70.6)\end{array}$ & .394 \\
\hline $\begin{array}{l}\text { Gender } \\
\text { Male } \\
\text { Female }\end{array}$ & $\begin{array}{r}395(27.3) \\
1,060(72.7)\end{array}$ & $\begin{array}{l}110(27.6) \\
356(33.6)\end{array}$ & $\begin{array}{l}288 .(72.4) \\
704(66.4)\end{array}$ & .030 \\
\hline $\begin{array}{l}\text { Marital status } \\
\text { Single } \\
\text { Partnered }\end{array}$ & $\begin{array}{r}425(28.9) \\
1,044(71.1)\end{array}$ & $\begin{array}{l}129(30.4) \\
339(32.5)\end{array}$ & $\begin{array}{l}296(69.6) \\
705(67.5)\end{array}$ & .429 \\
\hline $\begin{array}{l}\text { Employed } \\
\text { Yes } \\
\text { No }\end{array}$ & $\begin{array}{l}778(53.6) \\
674(46.4)\end{array}$ & $\begin{array}{l}250(32.1) \\
211(31.3)\end{array}$ & $\begin{array}{l}528(67.9) \\
463(68.7)\end{array}$ & .735 \\
\hline $\begin{array}{l}\text { Employment } \\
\text { Part-time } \\
\text { Full-time }\end{array}$ & $\begin{array}{l}526(68.6) \\
241(31.4)\end{array}$ & $\begin{array}{r}169(32.1) \\
83(34.4)\end{array}$ & $\begin{array}{l}357(67.9) \\
158(65.6)\end{array}$ & .527 \\
\hline $\begin{array}{l}\text { Retirement status } \\
\text { Yes } \\
\text { No }\end{array}$ & $\begin{array}{l}500(35.7) \\
899(64.3)\end{array}$ & $\begin{array}{l}151(30.2) \\
298(33.1)\end{array}$ & $\begin{array}{l}349(69.8) \\
601(66.9)\end{array}$ & .258 \\
\hline $\begin{array}{l}\text { Annual income } \\
\quad<\$ 50,000 \\
>\$ 50,000\end{array}$ & $\begin{array}{l}425(32.5) \\
884(67.5)\end{array}$ & $\begin{array}{l}114(26.8) \\
304(34.4)\end{array}$ & $\begin{array}{l}311(73.2) \\
580(65.6)\end{array}$ & .006 \\
\hline $\begin{array}{l}\text { Education } \\
\text { High school or less } \\
\text { College } \\
\text { Postgrad }\end{array}$ & $\begin{array}{l}349(24.1) \\
627(43.2) \\
475(32.7)\end{array}$ & $\begin{array}{r}95(27.2) \\
192(30.6) \\
175(36.8)\end{array}$ & $\begin{array}{l}254(72.8) \\
435(69.4) \\
300(63.2)\end{array}$ & .009 \\
\hline $\begin{array}{l}\text { Time in the United States } \\
\text { All other country } \\
\text { All my life }\end{array}$ & $\begin{array}{r}185(12.8) \\
1,259(87.2)\end{array}$ & $\begin{array}{r}46(24.9) \\
415(33.0)\end{array}$ & $\begin{array}{l}139(75.1) \\
844(67.0)\end{array}$ & .027 \\
\hline
\end{tabular}

Continued on next page 


\begin{tabular}{|c|c|c|c|c|}
\hline \multirow[b]{2}{*}{ Characteristic } & \multicolumn{3}{|c|}{ No. of patients, n (\%) } & \multirow[b]{2}{*}{$\boldsymbol{P}$} \\
\hline & $\begin{array}{c}\text { Total } \\
(\mathrm{N}=1, \mathbf{4 7 5})\end{array}$ & $\begin{array}{c}\text { Have } \\
\text { participated } \\
(n=470)\end{array}$ & $\begin{array}{c}\text { Have not } \\
\text { participated } \\
(n=1,005)\end{array}$ & \\
\hline $\begin{array}{l}\text { Race } \\
\text { Black } \\
\text { White } \\
\text { Hispanic } \\
\text { All Other races }\end{array}$ & $\begin{aligned} 73 & (5.0) \\
1,246 & (85.5) \\
31 & (2.1) \\
107 & (7.3)\end{aligned}$ & $\begin{aligned} & 16(22.0) \\
& 407(32.7) \\
& 4(12.9) \\
& 34(31.6)\end{aligned}$ & $\begin{array}{r}57(78.0) \\
839(67.3) \\
27(87.1) \\
73(66.3)\end{array}$ & .021 \\
\hline $\begin{array}{l}\text { Cancer type } \\
\text { Colon, rectal } \\
\text { Breast } \\
\text { Ovarian } \\
\text { Cervical, uterine } \\
\text { Prostate } \\
\text { Skin } \\
\text { Stomach } \\
\text { Pancreatic } \\
\text { Liver } \\
\text { Throat, esophageal } \\
\text { Kidney } \\
\text { Lung } \\
\text { Brain } \\
\text { Bone } \\
\text { Leukemia } \\
\text { Lymphoma } \\
\text { Bladder } \\
\text { Thyroid } \\
\text { Testicular } \\
\text { Multiple myeloma } \\
\text { Other }\end{array}$ & $\begin{array}{c}51(3.5) \\
566(38.4) \\
45(3.1) \\
23(1.6) \\
47(3.2) \\
41(2.8) \\
8(0.5) \\
10(0.7) \\
6(0.4) \\
24(1.6) \\
20(1.4) \\
64(4.3) \\
9(0.6) \\
13(0.9) \\
133(9.0) \\
184(12.5) \\
19(1.3) \\
24(1.6) \\
8(0.5) \\
69(4.7) \\
111(7.5)\end{array}$ & $\begin{array}{r}12(23.5) \\
199(35.2) \\
12(26.7) \\
6(26.1) \\
9(19.1) \\
13(31.7) \\
2(25.0) \\
3(30.0) \\
3(50.0) \\
5(20.8) \\
8(40.0) \\
19(29.7) \\
3(33.3) \\
8(61.5) \\
54(10.6) \\
48(26.1) \\
3(15.8) \\
3(12.5) \\
1(12.5) \\
18(26.1) \\
41(36.9)\end{array}$ & $\begin{array}{r}39(76.5) \\
367(64.8) \\
33(73.3) \\
17(73.9) \\
38(80.9) \\
28(68.3) \\
6(75.0) \\
7(70.0) \\
3(50.0) \\
19(79.2) \\
12(60.0) \\
45(70.3) \\
6(66.7) \\
5(38.5) \\
79(59.4) \\
136(73.9) \\
16(84.2) \\
21(87.5) \\
7(87.5) \\
51(73.9) \\
70(63.1)\end{array}$ & .020 \\
\hline $\begin{array}{l}\text { Initial diagnosis } \\
\text { 1950-1989 } \\
\text { 1990-1999 } \\
\text { 2000-2005 } \\
\text { 2006-April } 2013\end{array}$ & $\begin{array}{l}36(2.6) \\
139(10.2) \\
315(23.1) \\
871(64.1)\end{array}$ & $\begin{array}{r}12(33.3) \\
49(35.3) \\
128(40.6) \\
242(27.8)\end{array}$ & $\begin{array}{r}24(66.7) \\
90(64.7) \\
187(59.4) \\
629(72.2)\end{array}$ & .000 \\
\hline $\begin{array}{l}\text { Did not seek medical care } \\
\text { Yes } \\
\text { No/Not Sure }\end{array}$ & $\begin{array}{r}200(13.7) \\
1,265(86.3)\end{array}$ & $\begin{array}{r}72(36.0) \\
396(31.3)\end{array}$ & $\begin{array}{l}128(64.0) \\
869(68.7)\end{array}$ & .186 \\
\hline $\begin{array}{l}\text { Did not follow doctor's advice } \\
\text { Yes } \\
\text { Yes/Not Sure }\end{array}$ & $\begin{array}{r}203(13.9) \\
1,258(86.1)\end{array}$ & $\begin{array}{r}67(33.0) \\
400(31.8)\end{array}$ & $\begin{array}{l}136(67.0) \\
858(68.2)\end{array}$ & .732 \\
\hline $\begin{array}{l}\text { Trust oncologist } \\
\text { Disagree } \\
\text { Agree } \\
\text { Not sure }\end{array}$ & $\begin{aligned} 110 & (7.6) \\
1,273 & (87.8) \\
67 & (4.6)\end{aligned}$ & $\begin{array}{r}37(33.6) \\
409(32.1) \\
14(20.9)\end{array}$ & $\begin{array}{r}73(66.4) \\
864(79.1) \\
53(67.9)\end{array}$ & .142 \\
\hline
\end{tabular}

(29.6\%; $P=.018)$. Neutral attitudes toward being administered drugs during studies was significantly associated with higher patient participation $(35.6 \% ; P=.006)$. The majority of respondents, regardless of participation history, expressed unimportance concerning their desire for a cure (70.5\%) as a motivator for participation in clinical trials. This attitude was associated with participation in studies $(33.7 \% ; P=.01)$.

\section{Communication about clinical trials}

Respondents were also asked several questions regarding communication about clinical trials, including knowing others who have participated in trials, past sources of information about trials, attitudes about being approached about clinical trials, and information source preferences. Table 4 provides detailed information about each domain of questions. Most of the respondents (67.6\%) said they did not 
TABLE 2 Participants' attitudes about randomization

\begin{tabular}{|c|c|c|c|c|}
\hline \multirow[b]{2}{*}{ Question } & \multicolumn{3}{|c|}{ No. of patients, n (\%) } & \multirow[b]{2}{*}{$\boldsymbol{P}$} \\
\hline & $\begin{array}{c}\text { Total } \\
(N=1,475)\end{array}$ & $\begin{array}{c}\text { Have } \\
\text { participated } \\
(n=470)\end{array}$ & $\begin{array}{l}\text { Have not } \\
\text { Participated } \\
(n=1,005)\end{array}$ & \\
\hline $\begin{array}{l}\text { Okay with comparing different } \\
\text { treatments } \\
\text { Yes } \\
\text { No/Not Sure }\end{array}$ & $\begin{array}{l}801(54.8) \\
660(45.2)\end{array}$ & $\begin{array}{l}319(39.8) \\
148(22.4)\end{array}$ & $\begin{array}{l}482(60.2) \\
512(77.6)\end{array}$ & .000 \\
\hline $\begin{array}{l}\text { Okay with treatment chosen at } \\
\text { chance } \\
\text { Yes } \\
\text { No/Not Sure }\end{array}$ & $\begin{array}{l}562(38.4) \\
900(61.6)\end{array}$ & $\begin{array}{l}230(40.9) \\
236(26.2)\end{array}$ & $\begin{array}{l}332(59.1) \\
664(73.8)\end{array}$ & .000 \\
\hline $\begin{array}{l}\text { Treatment chosen at chance would } \\
\text { encourage your participation } \\
\text { Yes } \\
\text { No/Not Sure }\end{array}$ & $\begin{array}{c}268(18.3) \\
1,195(81.7)\end{array}$ & $\begin{array}{r}97(36.2) \\
369(30.9)\end{array}$ & $\begin{array}{l}171(63.8) \\
826(69.1)\end{array}$ & .091 \\
\hline $\begin{array}{l}\text { Knowing that it is okay to leave the } \\
\text { study if treatment did not suit you } \\
\text { would encourage your participation } \\
\text { Yes } \\
\text { No/Not Sure }\end{array}$ & $\begin{array}{c}1,022(70.0) \\
437(30.0)\end{array}$ & $\begin{array}{l}350(34.2) \\
113(24.4)\end{array}$ & $\begin{array}{l}672(65.8) \\
324(74.1)\end{array}$ & .002 \\
\hline $\begin{array}{l}\text { Knowing treatment options prior to } \\
\text { randomization would encourage } \\
\text { participation } \\
\text { Yes } \\
\text { No/Not Sure }\end{array}$ & $\begin{array}{c}1,000(68.9) \\
451(31.1)\end{array}$ & $\begin{array}{l}350(35.0) \\
115(25.5)\end{array}$ & $\begin{array}{l}650(65.0) \\
336(74.5)\end{array}$ & .000 \\
\hline $\begin{array}{l}\text { Knowing that either treatment was } \\
\text { suitable, that you could leave the } \\
\text { study, and that there was plenty of } \\
\text { information before the study would } \\
\text { encourage participation } \\
\text { Yes } \\
\text { No/Not Sure }\end{array}$ & $\begin{array}{l}992(68.7) \\
451(31.3)\end{array}$ & $\begin{array}{l}345(34.8) \\
109(24.2)\end{array}$ & $\begin{array}{l}647(65.2) \\
342(75.8)\end{array}$ & .000 \\
\hline $\begin{array}{l}\text { Randomization index } \\
\text { Zero positive } \\
1 \text { positive } \\
2 \text { positive } \\
3 \text { positive } \\
4 \text { positive } \\
5 \text { positive } \\
\text { All positive }\end{array}$ & $\begin{array}{l}217(15.5) \\
99(7.1) \\
160(11.4) \\
245(17.5) \\
248(17.7) \\
275(19.7) \\
154(11.0)\end{array}$ & $\begin{array}{r}46(21.2) \\
24(24.2) \\
42(26.2) \\
65(26.5) \\
83(33.5) \\
120(43.6) \\
62(40.3)\end{array}$ & $\begin{aligned} 171(78.8) \\
75(75.8) \\
118(73.8) \\
180(73.5) \\
165(66.5) \\
155(56.4) \\
92(59.7)\end{aligned}$ & .000 \\
\hline
\end{tabular}

know anyone else who had participated in a research study. Of those, $32.6 \% \mathrm{knew}$ if that person had a positive experience. Patients who knew someone who had participated in a research study were more likely to also have participated in a research study than not $(53.0 \%$ vs $21.7 \% ; P<$ $.001)$. About $61 \%$ of those who knew people with a positive research experience had participated in a research study, compared with nearly $27 \%$ participation among those whose contact did not have a positive experience $(P<.001)$.

Patients were asked about their previous sources of information concerning clinical trials. Oncologists (36.9\%), research staff (14.3\%) and websites (19.1\%) were the 3 most commonly identified sources of information. almost $32 \%$ of patients noted that they had never received information about research studies. Previous participation in a research study was highest in those who had been approached by research staff members (64.6\%) compared with those who were approached by an oncologist (54.7\%), clinic staff members $(54.6 \%)$, nurses $(59.6 \%)$, or primary care physicians $(42.6 \%)$, or those who had visited websites $(40 \%)$, received newsletters $(36.1 \%)$, or obtained other sources of information (43.4\%). 
TABLE 3 Attitudes regarding potential barriers and facilitators to participating in clinical trials

No. of patients, $\mathbf{n}(\%)$

\begin{tabular}{|c|c|c|c|c|}
\hline Potential barrier/facilitator & $\begin{array}{c}\text { Total } \\
(\mathrm{N}=1, \mathbf{4 7 5})\end{array}$ & $\begin{array}{c}\text { Have } \\
\text { participated } \\
(n=470)\end{array}$ & $\begin{array}{l}\text { Have not } \\
\text { Participated } \\
(n=1,005)\end{array}$ & $\boldsymbol{P}$ \\
\hline $\begin{array}{l}\text { Having weekend visits } \\
\text { Important } \\
\text { Unimportant } \\
\text { Neutral }\end{array}$ & $\begin{array}{l}376(29.2) \\
587(45.6) \\
323(25.1)\end{array}$ & $\begin{array}{l}112(29.8) \\
175(29.8) \\
113(35.0)\end{array}$ & $\begin{array}{l}264(70.2) \\
412(70.2) \\
210(65.0)\end{array}$ & .220 \\
\hline $\begin{array}{l}\text { Having evening visits } \\
\text { Important } \\
\text { Unimportant } \\
\text { Neutral }\end{array}$ & $\begin{array}{l}383(29.9) \\
595(46.4) \\
303(23.7)\end{array}$ & $\begin{array}{l}113(29.5) \\
183(30.8) \\
104(34.3)\end{array}$ & $\begin{array}{l}270(70.5) \\
412(69.2) \\
199(65.7)\end{array}$ & .378 \\
\hline $\begin{array}{l}\text { Being recommended by doctor } \\
\text { Important } \\
\text { Unimportant } \\
\text { Neutral }\end{array}$ & $\begin{array}{c}360(27.9) \\
891(69.0) \\
40(3.1)\end{array}$ & $\begin{array}{l}97(26.9) \\
293(32.9) \\
11(27.5)\end{array}$ & $\begin{array}{l}263(73.1) \\
598(67.1) \\
29(72.5)\end{array}$ & .107 \\
\hline $\begin{array}{l}\text { Length of study } \\
\text { Important } \\
\text { Unimportant } \\
\text { Neutral }\end{array}$ & $\begin{array}{l}359(27.8) \\
768(59.4) \\
166(12.8)\end{array}$ & $\begin{array}{l}102(28.4) \\
239(31.1) \\
63(38.0)\end{array}$ & $\begin{array}{l}257(71.6) \\
103(68.9) \\
529(62.0)\end{array}$ & .090 \\
\hline $\begin{array}{l}\text { Number of study visits } \\
\text { Important } \\
\text { Unimportant } \\
\text { Neutral }\end{array}$ & $\begin{array}{l}345(26.8) \\
765(59.5) \\
176(13.7)\end{array}$ & $\begin{array}{l}94(27.2) \\
239(31.2) \\
68(38.6)\end{array}$ & $\begin{array}{l}251(72.8) \\
526(68.8) \\
108(61.4)\end{array}$ & .029 \\
\hline $\begin{array}{l}\text { Research visits at the same time as } \\
\text { medical visits } \\
\text { Important } \\
\text { Unimportant } \\
\text { Neutral }\end{array}$ & $\begin{array}{l}356(27.7) \\
783(60.8) \\
148(11.5)\end{array}$ & $\begin{array}{c}90(25.3) \\
268(34.2) \\
44(29.7)\end{array}$ & $\begin{array}{l}266(74.7) \\
515(65.8) \\
104(70.3)\end{array}$ & .010 \\
\hline $\begin{array}{l}\text { Being compensated for travel } \\
\text { Important } \\
\text { Unimportant } \\
\text { Neutral }\end{array}$ & $\begin{array}{l}355(27.6) \\
595(46.3) \\
334(26.0)\end{array}$ & $\begin{array}{l}101(28.5) \\
176(29.6) \\
125(37.4)\end{array}$ & $\begin{array}{l}254(71.5) \\
419(70.4) \\
209(62.6)\end{array}$ & .018 \\
\hline $\begin{array}{l}\text { Not having drugs involved } \\
\text { Important } \\
\text { Unimportant } \\
\text { Neutral }\end{array}$ & $\begin{array}{l}340(27.0) \\
414(32.9) \\
503(40.0)\end{array}$ & $\begin{array}{l}102(30.0) \\
107(25.8) \\
179(35.6)\end{array}$ & $\begin{array}{l}238(70.0) \\
307(74.2) \\
324(64.4)\end{array}$ & .006 \\
\hline $\begin{array}{l}\text { Early access to new drugs } \\
\text { Important } \\
\text { Unimportant } \\
\text { Neutral }\end{array}$ & $\begin{array}{l}325(25.0) \\
812(62.5) \\
162(12.5)\end{array}$ & $\begin{array}{l}91(28.0) \\
272(33.5) \\
50(30.9)\end{array}$ & $\begin{array}{l}234(72.0) \\
540(66.5) \\
112(69.1)\end{array}$ & .191 \\
\hline $\begin{array}{l}\text { Money for participation } \\
\text { Important } \\
\text { Unimportant } \\
\text { Neutral }\end{array}$ & $\begin{array}{l}382(29.7) \\
483(37.5) \\
422(32.8)\end{array}$ & $\begin{array}{l}129(33.8) \\
138(28.6) \\
145(34.4)\end{array}$ & $\begin{array}{l}253(66.2) \\
345(71.4) \\
277(65.6)\end{array}$ & .120 \\
\hline $\begin{array}{l}\text { Better care/attention } \\
\text { Important } \\
\text { Unimportant } \\
\text { Neutral }\end{array}$ & $\begin{array}{l}325(25.2) \\
888(68.7) \\
79(6.1)\end{array}$ & $\begin{array}{c}91(28.0) \\
292(32.9) \\
27(34.2)\end{array}$ & $\begin{array}{c}234(72.0) \\
596(67.1) \\
52(65.8)\end{array}$ & .240 \\
\hline $\begin{array}{l}\text { Desire to help find a cure or } \\
\text { treatment } \\
\text { Important } \\
\text { Unimportant } \\
\text { Neutral }\end{array}$ & $\begin{array}{c}351(26.9) \\
919(70.5) \\
33(2.5)\end{array}$ & $\begin{array}{c}100(28.5) \\
310(33.7) \\
4(12.1)\end{array}$ & $\begin{array}{c}251(71.5) \\
609(66.3) \\
29(87.9)\end{array}$ & .010 \\
\hline
\end{tabular}




\begin{tabular}{|c|c|c|c|c|}
\hline \multirow[b]{2}{*}{ Potential barrier/facilitator } & \multicolumn{3}{|c|}{ No. of patients, n (\%) } & \multirow[b]{2}{*}{$\mathbf{P}$} \\
\hline & $\begin{array}{c}\text { Total } \\
(N=1,475)\end{array}$ & $\begin{array}{c}\text { Have } \\
\text { participated } \\
(n=470)\end{array}$ & $\begin{array}{c}\text { Have not } \\
\text { Participated } \\
(n=1,005)\end{array}$ & \\
\hline $\begin{array}{l}\text { Possible risk vs potential benefit } \\
\text { Important } \\
\text { Unimportant } \\
\text { Neutral }\end{array}$ & $\begin{array}{l}345(26.5) \\
900(69.2) \\
56(4.3)\end{array}$ & $\begin{array}{r}97(28.1) \\
294(32.7) \\
20(35.7)\end{array}$ & $\begin{array}{r}248(71.9) \\
606(67.3) \\
36(64.3)\end{array}$ & .240 \\
\hline $\begin{array}{l}\text { Discussing study with the oncologist } \\
\text { Important } \\
\text { Unimportant } \\
\text { Neutral }\end{array}$ & $\begin{array}{c}356(27.2) \\
939(71.7) \\
15(1.1)\end{array}$ & $\begin{array}{r}99(27.8) \\
314(33.4) \\
2(13.3)\end{array}$ & $\begin{array}{r}257(72.2) \\
625(66.6) \\
13(86.7)\end{array}$ & .046 \\
\hline $\begin{array}{l}\text { Discussing study with other cancer } \\
\text { center staff } \\
\text { Important } \\
\text { Unimportant } \\
\text { Neutral }\end{array}$ & $\begin{array}{c}323(24.7) \\
856(65.5) \\
127(9.7)\end{array}$ & $\begin{array}{r}93(28.8) \\
277(32.4) \\
44(34.6)\end{array}$ & $\begin{array}{r}230(71.2) \\
579(67.6) \\
83(65.4)\end{array}$ & .379 \\
\hline $\begin{array}{l}\text { Discussing study with family, friends, } \\
\text { and others with cancer } \\
\text { Important } \\
\text { Unimportant } \\
\text { Neutral }\end{array}$ & $\begin{array}{l}348(26.7) \\
807(61.9) \\
149(11.4)\end{array}$ & $\begin{array}{r}102(29.3) \\
258(32.0) \\
53(35.6)\end{array}$ & $\begin{array}{r}246(70.7) \\
549(68.0) \\
96(64.4)\end{array}$ & .372 \\
\hline $\begin{array}{l}\text { Knowing that I am well informed } \\
\text { about possible risks } \\
\text { Important } \\
\text { Unimportant } \\
\text { Neutral }\end{array}$ & $\begin{array}{c}357(27.4) \\
943(72.3) \\
4(0.3)\end{array}$ & $\begin{array}{r}97(27.2) \\
316(33.5) \\
1(25.0)\end{array}$ & $\begin{array}{r}260(72.8) \\
627(66.5) \\
3(75.0)\end{array}$ & .087 \\
\hline $\begin{array}{l}\text { Knowing it is okay to leave at any } \\
\text { time } \\
\text { Important } \\
\text { Unimportant } \\
\text { Neutral }\end{array}$ & $\begin{array}{l}350(26.9) \\
923(71.1) \\
26(2.0)\end{array}$ & $\begin{array}{r}97(27.7) \\
309(33.5) \\
6(23.1)\end{array}$ & $\begin{array}{r}253(72.3) \\
614(66.5) \\
20(76.9)\end{array}$ & .090 \\
\hline $\begin{array}{l}\text { Knowing that family/friends support } \\
\text { my decision to participate } \\
\text { Important } \\
\text { Unimportant } \\
\text { Neutral }\end{array}$ & $\begin{array}{l}362(27.9) \\
775(59.7) \\
162(12.5)\end{array}$ & $\begin{array}{r}103(28.5) \\
247(31.9) \\
63(38.9)\end{array}$ & $\begin{array}{r}259(71.5) \\
528(68.1) \\
99(61.1)\end{array}$ & .060 \\
\hline
\end{tabular}

Most respondents (84.1\%) believed that patients should be asked to take part in clinical trials, but only $69.9 \%$ were willing to be approached about clinical trials themselves. Acceptors to being approached were more likely to have participated in studies than those who were averse to being approached $(37.5 \%$ vs $18.3 \% ; P<.001)$. In addition, $93.6 \%$ of respondents indicated that they would like to receive information about research studies from their primary care physician, nurse practitioner, or oncologist. Almost $60 \%$ of patients wanted to receive information from the research coordinator, $37.5 \%$ from clinic staff, and $10 \%$ indicated that they preferred not to be approached at all.

\section{Discussion}

To our knowledge, this study is the first of its kind to be conducted across several different cancer care facilities and patient-support organizations in the state of New York. We recruited organizations that serviced patients in every region of the state. By recruiting participants from patient-support organizations, we were able to gain access to patients who may not have been actively in treatment or follow-up at the time of the study. We were also able to recruit patients with a range of cancer types and diagnoses. Ultimately, we were able to guide participants through the completion of the survey by using the existing staff, resources, and trained volunteers rather than having to rely on formal funding sources to cover facilitator costs.

We were able to recruit a sizeable sample through inperson and online survey methods, but we were not able to capture an accurate response rate because we used the 
TABLE 4 History of communication about clinical trials and preferences for future communication about clinical trials

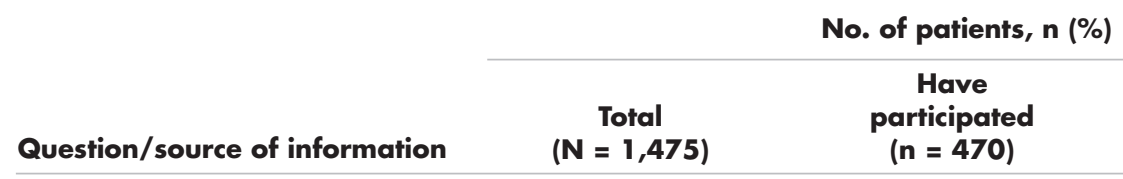

Communication with peers

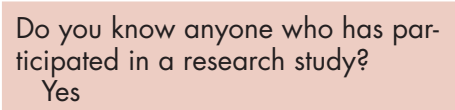

Did that person have a positive experience?

\begin{tabular}{|c|c|c|c|}
\hline $\begin{array}{l}\text { Yes } \\
\text { No }\end{array}$ & $\begin{array}{l}331(32.6) \\
684(67.4)\end{array}$ & $\begin{array}{l}203(61.3) \\
182(26.6)\end{array}$ & $\begin{array}{l}128(38.7) \\
502(73.4)\end{array}$ \\
\hline
\end{tabular}

Past sources of information

\begin{tabular}{|c|c|c|c|c|}
\hline $\begin{array}{l}\text { Primary-care physician } \\
\text { Yes } \\
\text { No }\end{array}$ & $\begin{array}{c}115(8.0) \\
1,328(92.0)\end{array}$ & $\begin{array}{r}49(42.6) \\
415(31.2)\end{array}$ & $\begin{array}{r}66(57.4) \\
913(68.8)\end{array}$ & .012 \\
\hline $\begin{array}{c}\text { Nurses } \\
\text { Yes } \\
\text { No }\end{array}$ & $\begin{aligned} 89 & (6.2) \\
1,354 & (93.8)\end{aligned}$ & $\begin{array}{r}53(59.6) \\
411(30.4)\end{array}$ & $\begin{array}{r}36(40.4) \\
943(69.6)\end{array}$ & .000 \\
\hline $\begin{array}{l}\text { Oncologist } \\
\text { Yes } \\
\text { No }\end{array}$ & $\begin{array}{l}532(36.9) \\
911(63.1)\end{array}$ & $\begin{array}{l}291(54.7) \\
173(19.0)\end{array}$ & $\begin{array}{l}241(45.3) \\
738(81.0)\end{array}$ & .000 \\
\hline $\begin{array}{l}\text { Research staff } \\
\text { Yes } \\
\text { No }\end{array}$ & $\begin{array}{r}206(14.3) \\
1,237(85.7)\end{array}$ & $\begin{array}{l}133(64.6) \\
331(71.3)\end{array}$ & $\begin{array}{r}73(35.4) \\
906(73.2)\end{array}$ & .000 \\
\hline
\end{tabular}

\begin{tabular}{|c|c|c|c|c|}
\hline $\begin{array}{l}\text { Clinic staff } \\
\text { Yes } \\
\text { No }\end{array}$ & $\begin{array}{c}108(7.5) \\
1,335(92.5)\end{array}$ & $\begin{array}{r}59(54.6) \\
405(30.3)\end{array}$ & $\begin{array}{r}49(45.4) \\
930(69.7)\end{array}$ & .000 \\
\hline $\begin{array}{l}\text { Newsletter } \\
\text { Yes } \\
\text { No }\end{array}$ & $\begin{array}{r}144(10.0) \\
1,299(90.0)\end{array}$ & $\begin{array}{r}52(36.1) \\
412(31.7)\end{array}$ & $\begin{array}{r}92(63.9) \\
887(68.3)\end{array}$ & .284 \\
\hline $\begin{array}{l}\text { Website } \\
\text { Yes } \\
\text { No }\end{array}$ & $\begin{array}{r}275(19.1) \\
1,168(80.9)\end{array}$ & $\begin{array}{l}110(40.0) \\
354(30.3)\end{array}$ & $\begin{array}{l}165(60.0) \\
814(69.7)\end{array}$ & .002 \\
\hline $\begin{array}{l}\text { Other sources of information } \\
\text { Yes } \\
\text { No }\end{array}$ & $\begin{array}{c}136(9.4) \\
1,308(90.6)\end{array}$ & $\begin{array}{r}59(43.4) \\
405(31.0)\end{array}$ & $\begin{array}{r}77(56.6) \\
903(69.0)\end{array}$ & .003 \\
\hline $\begin{array}{l}\text { None } \\
\text { Yes } \\
\text { No }\end{array}$ & $\begin{array}{l}458(31.7) \\
985(68.3)\end{array}$ & $\begin{array}{c}26(5.7) \\
438(44.5)\end{array}$ & $\begin{array}{l}432(94.3) \\
547(55.5\end{array}$ & .000 \\
\hline & Commun & ut recruitment & & \\
\hline $\begin{array}{l}\text { Do you think that patients should } \\
\text { be asked to take part in research } \\
\text { studies? } \\
\text { Yes } \\
\text { No/Not Sure }\end{array}$ & $\begin{array}{r}1,229(84.1) \\
233(15.9)\end{array}$ & $\begin{array}{r}443(36.0) \\
24(10.3)\end{array}$ & $\begin{array}{l}786(64.0) \\
209(98.7)\end{array}$ & .000 \\
\hline
\end{tabular}




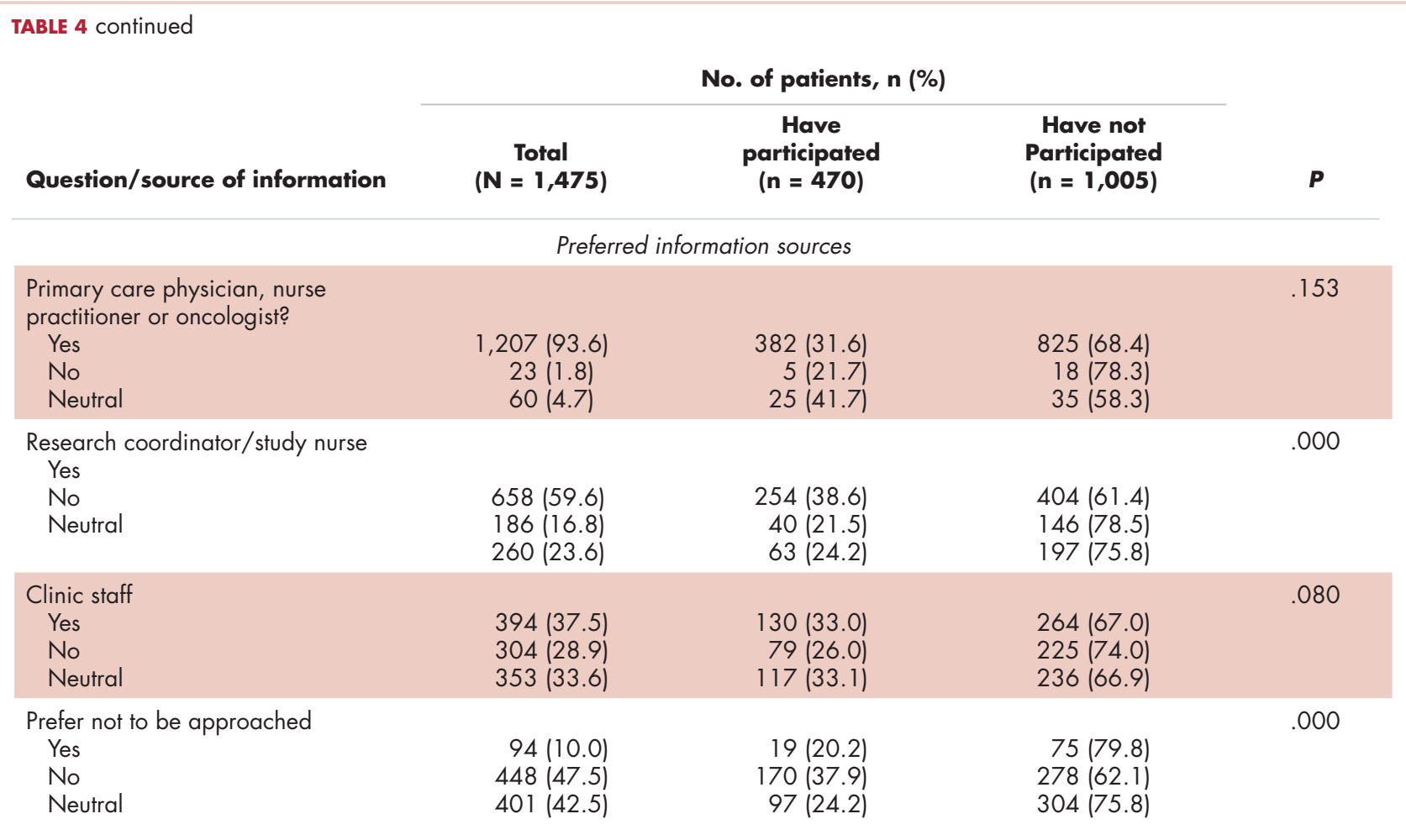

organizations' listservs and did not know how many people had received the invitation to participate and declined (compared with e-mail bounce-back). We know that at the sites where people were approached in waiting areas, very few people declined to participate. Our findings are largely limited to responses of well-educated, middle- to high- income households, and white women. Compared with national and New York state incidence data, ${ }^{15,16}$ breast and hematologic cancers were overrepresented, and other cancers such as colorectal cancer were underrepresented in this sample. Oversampling is in part the result of recruiting through some cancer-specific sites (ie, a breast cancer clinic at one facility and a hematologic cancer-specific patient-support organization). Efforts were made to reach public hospitals and Federally Qualified Health Centers, which would likely have had a larger low-income population, but we were not successful in garnering a champion to administer and manage the survey at those institutions. Recruitment of a demographically diverse sample is not restricted to this study, but is true for recruitment to many other trials as well. Having a more representative sample of participants strengthens the generalizability of the findings and supports future treatments and procedures for all populations. Our recruitment methods tapped into an existing patient base (those in treatment waiting rooms and belonging to existing e-mail lists) of the recruiting organizations. If we had reached a truly representative sample of cancer patients in these organizations, then the challenge may be to increase the diversity of the patient population of organizations that service cancer patients. This will require a multilevel approach that not only will involve staff responsible for recruitment into trials, but also an institution-wide support of community outreach to increase the diversity of an organization's patient population.

We found that almost $32 \%$ of our respondents had never spoken with anyone or read anything about clinical trials, despite the evidence supporting communication about clinical trials with patients. ${ }^{6,10,11} \mathrm{It}$ is possible that patients were not told about clinical trials because there were no trials available for their type or stage of cancer, but we did not assess that. It is also possible that recall bias may also have influenced these findings, especially in those who were diagnosed a longer time before (eg, 1950 through 1980). We encountered many of the same barriers to clinical trial participation that have been previously reported in the literature, including concern about randomization, $3,4,6,7$ inconvenience of the study, ${ }^{4}$ and use of novel drugs in a trial. ${ }^{7}$ Those who had previously participated in a trial had greater acceptance about various elements of randomization. Those who expressed neutral attitudes about either the number of visits required for a trial, being compensated for participation, or the involvement of drugs in a trial, were also more likely to have reported previous participation in a trial.

On the basis of our findings, we pose the following lessons learned and suggestions to improve recruitment into 
clinical trials. First, a team approach to educating patients about clinical trials and specific opportunities within their institution may increase trial awareness in cancer patients. Although patients in our study said they preferred to receive information from their oncologist, their participation in a study may also be associated with information received from research staff. Communication about clinical trials does not have to be limited to the responsibility of the oncologist. Other physicians, nurses, and research staff can be effective sources of information for patients as they make decide about participating in clinical trials. Baer and colleagues, for example, provide recommendations on how to engage referring physicians in communication about trials. ${ }^{17}$

Second, conversations with health care providers and study staff should continue to focus on well-established barriers to participation. Randomization continues to be a concept that influences participation in research studies and should be a barrier addressed in meetings with patients. Patients may feel more comfortable and more open to enrollment with an in-depth and a clearly explained description of randomization. ${ }^{6}$

Third, trial recruitment has traditionally emphasized either compensation or a desire to help find a cure to encourage participation in studies. These facilitators may not be important to participants and may not need to be communicated. And fourth, encouraging opportunities for past clinical trial participants to speak with those considering trials may have an impact on a patient's decision to participate. Those in our study who knew someone who had a positive experience in a clinical trial were more likely to have participated in a trial themselves. Incorporating previous trial participants or their testimonies into education interventions may be a way to facilitate information sharing about positive experiences.

More in-depth exploration is needed to understand why patients may differentiate between feelings that, in general, patients should be approached about research studies, but that they themselves are less willing to participate in a clinical trial.

\section{Acknowledgments}

The authors acknowledge the support of the New York State Cancer
Consortium. They thank the staff and volunteers at the organizations that aided with participant recruitment, administered the surveys, and provided online survey support. In particular, they thank Rachel Jordan for developing the questions, Jennifer John and Michael Maley for administering the survey in the outpatient clinics, and Rishi Sheth and Neha Qzai for help with data entry and cleaning.

\section{References}

1. National Institutes of Health, US Department of Health and Human Services. National Cancer Institute, Clinical Trials. http:// www.cancer.gov/clinicaltrials/search. Accessed January 16, 2015.

2. Murthy VH, Krumholz HM, Gross CP. Participation in cancer clinical trials. JAMA. 2004;291:2720-2726.

3. Mills EJ, Seely D, Rachlis B, et al. Barriers to participation in clinical trials of cancer: a meta-analysis and systematic review of patient-reported factors. Lancet Oncol. 2006;7:141-148.

4. Schmotzer GL. Barriers and facilitators to participation of minorities in clinical trials. Ethn Dis. 2012;22:226-230.

5. Unger JM, Hershman DL, Albain KS, et al. Patient income level and cancer clinical trial participation. J Clin Oncol. 2013;31:536-542.

6. Jenkins V, Farewell D, Batt L, et al. The attitudes of 1066 patients with cancer toward participation in randomised clinical trials. Br J Cancer. 2010;103:1801-1807.

7. Jenkins V, Farewell V, Farewell D, et al. Drivers and barriers to patient participation in RCTs. Br J Cancer. 2013;108:1402-1407.

8. Meropol NJ, Buzaglo JS, Millard J, et al. Barriers to clinical trial participation as perceived by oncologists and patients. J Natl Compr Canc Netw. 2007;5:655-664.

9. Ford JG, Howerton MW, Bolen S, et al. Knowledge and access to information on recruitment of underrepresented populations to cancer clinical trials. Evid Rep Technol Assess (Summ). 2005;122:1-11.

10. Fenton L, Rigney M, Herbst RS. Clinical trial awareness, attitudes, and participation among patients with cancer and oncologists. Commun Oncol. 2009;6:207-213,228.

11. Weckstein DJ, Thomas CA, Emery IF, et al. Assessment of perceived cost to the patient and other barriers to clinical trial participationJ Oncol Pract. 2011;7:330-333.

12. New York State Cancer Consortium. 2012-2017 New York State comprehensive cancer control plan. http://www.nyscancerconsortium.org/documents/NYSCompCancerPlan2012-2017-FINAL.pdf. Updated 2012. Accessed January 16, 2015.

13. Comis RL, Miller JD, Colaizzi DD, Kimmel LG. Physician-related factors involved in patient decisions to enroll onto cancer clinical trials. J Oncol Pract. 2009;5:50-56.

14. Anderson LA, Dedrick RF. Development of the Trust in Physician Scale: a measure to assess interpersonal trust inpaitient-physician relationships. Psychol Rep. 1990;67:1091-1100.

15. American Cancer Society. Cancer facts \& figures 2014. http://www. cancer.org/acs/groups/content/@research/documents/webcontent/ acspc-042151.pdf. Accessed January 16, 2015.

16. New York State Cancer Registry. Cancer incidence and mortality in New York state, 1976-2011. http://www.health.ny.gov/statistics/cancer/registry/. Updated March 2014. Accessed March 13, 2014.

17. Baer AR, Michaels M, Good MJ, Schapira L. Engaging referring physicians in the clinical trial process. J Oncol Pract. 2012;8(1):e8-e10. 